\title{
Une analyse log-linéaire des courants migratoires interprovinciaux : Canada, 1961-1983
}

\section{A Log-linear Analysis of Interprovincial Streams of Migration:}

Canada, 1961-1983

\section{Un análisis logo-lineal de los flujos migratorios interprovinciales: Canadá, 1961-1983}

\section{Jacques Ledent}

Volume 12, numéro 2, octobre 1983

Les migrations

URI : https://id.erudit.org/iderudit/600508ar

DOI : https://doi.org/10.7202/600508ar

Aller au sommaire du numéro

Éditeur(s)

Association des démographes du Québec

ISSN

0380-1721 (imprimé)

1705-1495 (numérique)

Découvrir la revue

Citer cet article

Ledent, J. (1983). Une analyse log-linéaire des courants migratoires interprovinciaux : Canada, 1961-1983. Cahiers québécois de démographie, 12(2), 223-250. https://doi.org/10.7202/600508ar
Résumé de l'article

Cet article présente une analyse approfondie des caractéristiques spatio-temporelles de la migration interprovinciale au Canada réalisée sur la base de données longitudinales tirées du fichier des allocations familiales. La méthodologie utilisée fait appel au modèle log-linéaire sous-jacent à la notion de tableau de contingence, lequel modèle autorise une représentation simple des données observées à l'aide de paramètres caractéristiques des diverses interactions possibles entre les variables concernées (variables d'origine, de destination et de temps dans le cas présent). Dans une première étape, un examen comparé de la performance des diverses variantes non saturées (variantes où manquent un ou plusieurs des paramètres pertinents) permet de clarifier l'influence globale de chacune des variables. Dans une deuxième étape, cette influence est précisée à l'aide d'une investigation détaillée des paramètres relatifs à la variante de quasi-indépendance, c'est-à-dire, la variante où seul l'effet d'interaction s'exerçant entre les trois variables est ignoré. Outre la suggestion que les variables d'origine et de destination ne sont pas indépendantes (résultat qui confirme la nécessité de mettre l'accent sur les courants migratoires dans l'étude de la migration), cette investigation met en avant quelques conclusions sur les variations temporelles de la migration interprovinciale. 
Cahiers québécois de démgraphie

Vol. 12, no 2 , octobre 1983

\title{
Une analyse log-linéaire des courants migratoires interprovinciaux: Canada, 1961:1983
}

\author{
Jacques LEDENT*
}

\section{INTRODUCTION}

L'etude de la migration interrégionale donne généralement lieu a des analyses en coupe transversale, car les donnés pertinentes aisement accessibles au chercheur sont presque toujours des donnés en provenance d'un recensement de population. Mais, a une epoque où se manifeste une demande sans cesse croissante pour des perspectives démographiques multirégionales, de telles analyses ne permettent plus de subvenir aux besoins de maniere satisfaisante.

En effet, la preparation de perspectives démographiques plus réalistes que celles produites a ce jour par les bureaux statistiques nationaux réclame une bonne comprehension du rôle joué par la variable temps dans le processus migratoire. Malheureusement, les analyses de donnees transversales $n$ autorisent pas une telle compréhension meme si, dans certains cas favorables, la disponibilité de données tirées de deux ou plusieurs recensements successifs est susceptible, au moyen d'une approche de statique comparee, de fournir quelques indications a ce sujet. Aussi l'étude de la migration interrégionale doit-elle, dans la mesure du possible, s'orienter vers la réalisation d'analyses basées sur des données longitudinales.

Une telle orientation s'observe deja dans plusieurs pays disposant d'un registre de population, notamment la Suede et les Pays-Bas, car la compilation d'un registre de population, pour une période quelconque, autorise l'établissement de la matrice des courants migratoires origine-destination en relation avec tout découpage d'interet. L'on sait qu'au Canada il n'existe pas de registre de population; mais, dans le cas où l'on s'interesse a la migration interprovinciale, il existe une source de remplacement, a savoir le fichier des

* Institut national de la recherche scientifique - Urbanisation, Université du Québec, Montréal.

L'auteur tient à exprimer une reconnaissance particulière à Frans Willekens et Nazli Baydar qui, lors d'un court sejour a N.I.D.I. (Netherlands Interuniversity Demographic Institute), l'ont aiguillé vers l'utilisation du modele log-linéaire comme outil d'analyse pertinent dans l'étude de la migration interrégionale. 
allocations familiales ${ }^{2}$ a partir duquel statistique Canada établit l'intensité mensuelle des courants origine-destination. Quoique disponible depuis prés d'une décennie ${ }^{2}$, cette source n'a pas encore, a notre connaissance, été utilisée pour sous-tendre une analyse pertinente de type longitudinal.

Lobjectif de cet article est de pallier cet état de fait en examinant de maniere approfondie la variation annuelle des courants migratoires interprovinciaux. A cet effet, l'on fait appel a une approche encore peu utilisée a ce jour mais dont l utilisation sest révélée trés prometteuse dans une analyse de la stabilité temporelle de la migration aux Fays-Eas (Baydar, 1983). Initialement suggéree et développée par willekens (1982, 1983a et 1983b), cette approche repose sur le modele log-linéaire typique de l'analyse de donnés catégoriques croisées, ou analyse de contingence, fréquemment rencontrée dans le domaine de la statistique.

En bref, le modele log-linéaire est un outil statistique adapté à l'étude de structures complexes qui permet de souligner l'influence de tous les elements entrant en jeu. Ainsi son application a letude des courants migratoires interrégionaux Mat mene-t-elle a lexamen du róle joué par la variable temps $t$ mais aussi de ceux joues par les autres éléments intervenants, cest-a-dire la région d'origine i et la région de destination j. De fait, les divers éléments concernés ne sont pas considérés de manière separée mais plutót au travers des interactions s'exercant entre eux. Il s'ensuit que le modele log-linéaire s'annonce comme une des techniques les plus appropries pour mener a bien dans un cadre unique une étude revelatrice des aspects spatio-temporeis de la migration.

Ci-apres, l application de ce modele aux donnés annuelles de la migration interprovinciale canadienne sur la periode 1 er juin 1961 - 31 mai 1983 (5oit 22 annés fiscales) fait l'objet de quatre sections. Apres un bref rappel de la thérie et de la pratique du modele log-linéare, la section 1 tente d'évaluer l'influence globale des trois élements intervenants soulignes plus haut: origine, destination et temps. Afin de préciser cette influence, la section 2 propose une analyse plus détaillée qui s'appuie sur une variante particuliere (variante dite de quasi-independance) du modele préconisé. La discussion des résultats initiée des cette section est poursuivie dans les deux suivantes, ou l'on s'attache a caractériser de manière significative l influence des variables temps (section 3 ) et espace (section 4 ).

1 Depuis peu, le chercheur a également accés à une deuxieme source, le fichier des impots, laquelle source présente lavantage d'offrir une couverture spatiale plus large: voir Norris, 1983.

2 Statistique Canada a publié dans la premiere livraison (en 1977) de son catalogue 91-208 intitulé higrations internationales et interprovinciales au Canada la matrice consolidée des courants interprovinciaux pour chacune des années fiscales de la période 1961-1962 a 1975-1976, puis, dans chaque nouvelle livraison annuelle, la matrice correspondant à l'année fiscale écoulée. 


\section{INFLUENCE GLOBALE DES ÉLÉMENTS INTERVENANTS}

Le modele log-linéaire met en jeu un certain nombre de parametres qui chacun refletent la contribution a l'influence d'ensemble des effets associés aux diverses interactions possibles existant entre les élements considerés. Dans le cas présent, il implique trois éléments:

- 1 élement $A$, ou origine de chaque courant migratoire, pour lequel il existe R catégories (autant que de provinces): $i=1, \ldots, f$

- l élement $B$, ou destination de chaque courant migratoire, pour lequel il existe egalement $k$ categories: $j=1, \ldots, k$

- l élément $[$, ou période a laquelle est associé chaque courant migratoire, pour lequel il existe $T$ categories: $t=1$ (annee fiscale $1961-1962$ ), ... T (année fiscale 1982-1983).

Dans ces conditions, le courant migratoire de la province i vers la province $j$ au temps $t$ peut $s$ exprimer à l'aide de

$M_{1, t}=W W_{1}^{A} W_{j}^{B} W_{t}^{C} W_{1 j}^{A D} W_{1 t}^{A C} W_{j t}^{B C} W_{1 j t}^{A B C}$

Les différents termes apparaissant dans cette équation réflètent les divers effets possibles 1 iant les trois éléments considerés:

a) w represente un effet géneral moyen

b) $w_{1}^{A}, w_{j}$ et $w_{t}^{c}$ représentent les effets principaux associes aux variables $i, j$ et $t$ respectivement

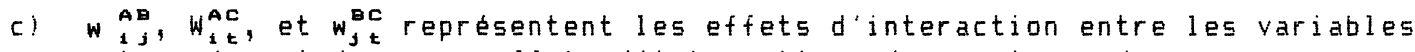
prises deux à deux, ou effets d'interactions du premier ordre

d) Wac représente 1 effet d'interaction entre les 3 variables considerées, ou effet d'interaction du deuxième ordre.

Au passage, I on notera qu'en égalant les valeurs logarithmiques des deux membres de 1 équation (1) l on obtient l'expression suivante, laquelle justifie pleinement le nom de modele log-linéaire utilisé plus haut:

$\ln M_{i j t}=u+u_{1}^{A}+u_{j}^{B}+u_{t}^{C}+u_{i j}^{A B}+u_{1 t}^{A C}+u_{j t}^{B C}+u_{i j t}^{A B C}$

Ainsi donc, le modele ici consideré se presente-t-il sous deux versions equivalentes, l une multiplicative (équation l) et l'autre additive (equation 2). Vu sa relation avec le modele classique de gravité (pour une revue de ce modele, voir hua et Forel1, 1979), la premiere formulation est particulierement adapté a l'etude de tout phénomene d'interaction spatiale; dou son utilisation dans le present article. Far contre, en raison d'une parenté evidente au niveau du paradigme sous-jacent avec l analyse de variance (ANOVA) classique, la deuxieme formulation est plus utilisé dans le domaine de la statistique.

Cette dernière parenté nous conduit d'ailleurs a indiquer ici que chacun des termes apparaissant dans l'expression (1) lou alternativement l'expression 
(2)) ne représente qu une déviation par rapport a la moyenne géometrique (algebrique) des termes du mene type. Ainsi, les divers parametres apparaissant dans (1) vérifient-ils les relations suivantes:

$$
\begin{aligned}
& \pi w_{1}^{A}=\pi \quad w_{j}^{B}=\pi w_{t}^{C}=1
\end{aligned}
$$

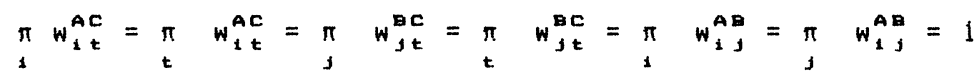

$$
\begin{aligned}
& \pi W_{1 j t}^{A B C}=\pi \quad W_{1 j t}^{A B C}=\pi \quad W_{1 J t}^{A B C}=1
\end{aligned}
$$

Si. tous les termes figurant dans l'equation (1) sont effectivement inclus dans la description de $M_{1}$, , la variante du modele log-lineaire alors considérée est dite saturée. Mais il se peut que les effets d'interaction entre variables ne soient pas tous significatifs au point que la presence de certains des parametres dans la description de $M_{1 j t} 5$ avere inutile. En ce cas, qu'il manque un ou plusieurs parametres, la variante résultante est dite non saturée.

En pratique, une analyse comparee des variantes non saturés ${ }^{3}$ permet d'eclairer l'impact des différentes variables impliquees, c'est-a-dire, les provinces dorigine et de destination et le temps. A cet effet, l'ensemble des variantes non saturees est mis en oeuvre par l'application d'un programme approprié -- sous-routine PJF de la bibliotheque BMDP (Dixon et Brown, 1979) -aux donnés migratoires mentionnés plus haut. De façon précise, pour une variante donnée, ce programme détermine la valeur des courants estimes ${ }^{4}$ puis calcule le logarithme du ratio de vraisemblance

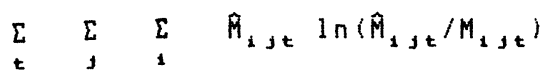

un indice qui reflete la capacité de la variante a générer des valeurs estimés $\left(\hat{A}_{1 \mathrm{t}}\right.$ ) qui soient aussi proches que possible des valeurs observés correspondantes $\left(M_{1}, t\right)$.

3 Cette analyse est limitee a la consideration des variantes de type hiérarchique. En d'autres termes, si un parametre quelconque est ignoré, les parametres d'ordre superieur dont les variables associés comprennent celle(s) associée(s) au parametre ignoré doivent l'etre également.

4 Lon renvoie le lecteur a Fienberg (1977: 32-34) pour une discussion de la méthode de calcul de ces valeurs, car sa presentation n'est pas indispensable pour la compréhension du reste de cette section. 
Le tableau 1 montre 1 a valeur de cet indice pour chacune des 17 variantes possibles. De la comparaison des valeurs relatives aux variantes 1,2 et 5 , il ressort une certaine préminence des variables spatiales -- la province de destination ayant cependant une plus grande influence que la province dorigine - sur la variable de temps. Ce résultat est d'ailleurs renforcé par le faible accroissement de performance qu'entraine la consideration de la variable de temps a coté de la province d'origine (voir variantes du groupe B) ou celle de destination (voir variantes du groupe $C$ ) ou bien meme les deux (voir variantes du groupe D).

L'importance de la dimension spatiale est par ailleurs soulignée par:

1) Ia diminution trés substantielle enregistrée par l'indice d'écart entre valeurs observees et estimees quand les variables diorigine et de destination sont considerées simultanement (variante 8 ) au lieu de separement (variantes 2 et 5 )

2) le nouveau gain important de réalisme qu'apporte l'inclusion de l'effet d'interaction entre les deux variables spatiales (variante 13) par rapport a la seule considération simultanée de ces variables (variante 8 ).

TABLEAU 1

Indice décart entre courants estimés et observés (logarithme du ratio de vraisemblance) pour chaque variante non-saturée

\begin{tabular}{|c|c|c|c|}
\hline Groupe & Variante & Parametres & Indice d'écart \\
\hline A. & 1 & $t$ & 13265 \\
\hline B. & $\begin{array}{l}2 \\
3 \\
4\end{array}$ & $\begin{array}{l}i \\
i, t \\
i t\end{array}$ & $\begin{array}{ll}9 & 927 \\
9 & 656 \\
9 & 496\end{array}$ \\
\hline C. & $\begin{array}{l}5 \\
6 \\
7\end{array}$ & $\begin{array}{l}j \\
j, t \\
j t\end{array}$ & $\begin{array}{ll}8 & 669 \\
8 & 598 \\
8 & 366\end{array}$ \\
\hline D. & $\begin{array}{r}8 \\
9 \\
10 \\
11 \\
12\end{array}$ & $\begin{array}{c}i, j \\
i, j, t \\
i t, j \\
i, j t \\
i t, j t\end{array}$ & $\begin{array}{ll}4 & 001 \\
3 & 931 \\
3 & 770 \\
3 & 698 \\
3 & 526\end{array}$ \\
\hline E. & $\begin{array}{l}13 \\
14 \\
15 \\
16 \\
17\end{array}$ & $\begin{array}{c}i j \\
i j, t \\
i j, i t \\
i j, j t \\
i j, i t, j t\end{array}$ & $\begin{array}{l}638 \\
567 \\
406 \\
335 \\
149\end{array}$ \\
\hline
\end{tabular}


Cette double observation peut légitimement s'interpreter comme un rejet de l'hypothese d'independance entre les régions d'origine et de destination sous-jacente aux perspectives demographiques multireqionales réalisés par le Bureau du recensement des Etats-Unis (US Eureau of Census, 1967) et Statistique Canada (1979).

Enfin, l observation des indices d'ecart relatifs aux variantes du groupe $E$ suggère l'influence décisive de la variable temps une fois les élements spatiaux convenablement pris en compte. Ce résultat peut s'interpreter comme la manifestation dune certaine instabilité temporelle au niveau de l'impact de la province d'origine et surtout de la province de destination.

\section{VERS UNE ANALYSE DÉTAILLÉE DES ÉLÉMENTS INTERVENANTS}

Afin de préciser l'influence globale des variables d'espace et de temps mises en évidence ci-dessus, un examen et une interpretation des parametres relatifs a la variante saturée apparaít de mise. Lorsque les donnés migratoires disponibles incluent les nombres de migrations intrarégionales, cette tache ne pose guere de difficultes: voir Baydar (1983) pour une illustration sur la base de donnees de migration inter- et intraprovinciales aux Pays-Bas. En effet, les valeurs des parametres relatifs aux divers effets sobtiennent facilement a partir des formules ci-dessous (Willekens, 1983b: 247):

- effet general moyen:

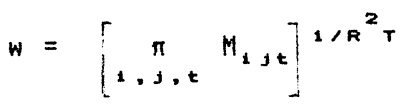

- effets principaux:

$w_{i}^{A}=\frac{1}{w}\left[\begin{array}{cc}\pi & M_{1 j t} \\ j, t & 1 / R T\end{array}\right.$

$w_{j}$ et $w_{t}^{c}$ : formules analogues

- effets dinteraction du premier ordre:

$$
\begin{aligned}
& W_{1 S}^{A B}=\frac{1}{y}\left[\begin{array}{ll}
\pi & M_{1 j t} \\
t & 1 / T
\end{array}\right. \\
& \text { avec } y=w w_{1}^{A} w_{j}^{D} w_{t}^{c} \\
& \text { We et woc : formules analogues } \\
& w_{1 j t}^{A D C}=\frac{1}{2} M_{1 j t} \\
& \operatorname{avec} z=w w_{1}^{A} w_{j}^{D} w_{t}^{C} w_{1 j}^{A D} w_{1 t}^{A C} w_{j t}^{D C}
\end{aligned}
$$


Far contre, dans le cas où les nombres de migrations intraregionales sont inconnus (comme tel est presentement le cas), la tache du chercheur se complique quelque peu. En effet, le tableau des donnés migratoires comporte des entrés nécessairement nulles, ou zéros structurels, qui ont pour conséquence dinvalider les formules signalees ci-dessus. Suite a une suggestion de Bishop, Fienberg et Holland (1775, chapitre 5), Willekens (1983a: 201) propose de parer a cette éventualite par l'utilisation des memes formules avec suppression pure et simple des entrées nulles. Dans ce cas fifi-1) $5 e$ substitue a $F^{2}$ au niveau de l exposant apparaissant dans l'équation relative à 1 'effet général moyen et (R-1) se substitue a $F$ au niveau de 1 'exposant apparaissant dans les équations relatives aux effets principaux. Cette proposition a cependant l'inconvenient de ne pas satisfaire l ensemble des restrictions (3a) - (3c) (Eishop, Fienberg et Holland, 1975: 211 ) et donc de rendre difficile l'interpretation des parametres.

Néanmoins, le contexte particulier de cette étude cou la variable temps sajoute aux variables dorigine et de destinationl autorise la consideration d'une solution de remplacement qui, comme on le verraplus loin, s'avere trés satisfaisante. L'idée est ici de faire appel a la variante non saturée dite variante de quasi-independance, oú seul manque l'effet d'interaction du second ordre. Dans ces conditions, le modele log-linéaire devient

$$
\hat{M}_{1 j t}=W W_{1}^{A} w_{j}^{B} W_{t}^{C} W_{i j}^{A B} W_{1 t}^{A C} W_{j t}^{B C}
$$

ou le chapeau ajouté au symbole $M$ des courants migratoires indique qu'il 5 'agit

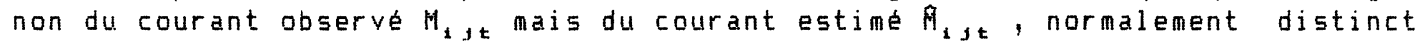
du précedent.

En pratique, l'estimation des parametres figurant a la partie droite de (5) requiert, comme préalable, la détermination des courants estimés Mijt. Fort heureusement, cette dernière détermination ne pose guere de problèmes. En effet, 1 ensemble $\hat{A}$ des courants $\hat{M}_{1 \text { it }}$ est un ensemble d'éléments auxquels sont i mposés

- une structure prédéterminée des effets d'interaction du deuxieme ordre

$$
\text { ( } \left.w_{i, t}^{A B C}=15 i j \neq i, w_{1, t}^{A B C}=05 i j=i\right) \text { et }
$$

- un jeu de contraintes marginales issues de l'utilisation de la methode de vraisemblance (Fienberg, 1977: 37 )

$$
\begin{aligned}
& \underbrace{}_{t} \hat{H}_{1, t}=\sum_{t} M_{1 j t} \\
& \sum M_{1 j t}=\sum M_{1 j t} \\
& \sum B_{1 j t}=\sum M_{1 j t}
\end{aligned}
$$


Lon est ainsi amené a utiliser, pour l'estimation de cet ensemble, une méthode iterative généralisant au cas de trois dimensions la methode RAs utilisée en particulier pour l'actualisation des tableaux déchanges interindustriels. Initialement, on definit une premiere valeur de M,

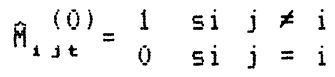

Puis, pour $v=0$, on calcule successivement

a) $m_{1 j t}(3 v+1)=\frac{\sum M_{1 j t}}{\sum_{t} m_{1 j t}(3 v)}$

de sorte que les valeurs estimés satisfassent les contraintes marginales (ba)

b) 角ijt $(3 v+2)=\frac{\sum H_{i j t}}{\sum h_{1 j t}(3 v+1)}$

de sorte que les valeurs estimés satisfassent les contraintes marginales (bb)

c) $\left.M_{1 j t} i 3 v+3\right)=\frac{\sum M_{1 j t}}{\sum M_{1 j t}(3 v+2)}$

de sorte que les valeurs estimés satisfassent les contraintes marginales (bc).

Bien entendu, l'imposition de ces dernieres contraintes annule la validité des contraintes précedentes. L'on répete donc le cycle (8a) - (8c) pour $v=1,2, \ldots$ jusqu a ce que les valeurs de $\hat{A}_{1 \text { it }}$ demeurent virtuellement inchangés d'un cycle a l'autre, car cette procédure connue sous le nom de procedure itérative de calibrage proportionnel (Deming and $5 t e p h a n, 1940$ ) converge nécessairement.

Enfin, une fois les valeurs estimés H $_{\text {j }}$ obtenues, les divers parametres apparaissant dans la partie droite de (5) s obtiennent tres simplement par application des formules presentées en annexe.

Passant maintenant a l'examen des parametres ainsi déterminés, 1 'on 5 intéresse tout d'abord au parametre w representatif de l'effet general moyen. $L$ on se souvient (voir équation 1) que si la structure étudiée ne comporte pas de zéros structurels, l'effet général moyen associé a la variante saturée représente la moyenne géométrique de l'ensemble des observations. Dans le cas présent (variante de quasi-independance avec $\hat{H}_{1 j t}=0$ pour $j=i$ ), il est facile de montrer, a partir de (5), que

$$
W=\left[\begin{array}{cc}
\pi & R_{1}, t \\
1,1, t & 1 / R(R-1) T
\end{array}\right]^{1 / 2}
$$


de sorte que leffet genéral moyen represente la moyenne géométrique des courants estimés. Empiriquement, lon trouve que $w=1503,7$ alors que la moyenne géometrique des courants (non nuls) observés est égale $₫ 1457,0$.

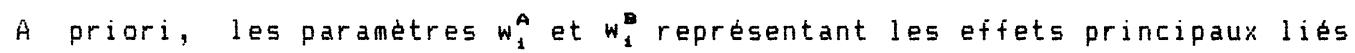
a lorigine et a la destination peuvent s'interpréter comme des indices composites refletant respectivement les forces de répulsion et dattraction associées a chacune des provinces. Les valeurs prises par ces parametres, fournies a la partie gauche du tableau 2 , sont, au niveau de chacune des provinces, trés proches. De fait, il existe une forte corrélation entre wi et $w_{1}$ (le coefficient de corrélation 5 etablit a 0,983 ) qui tend a valider, dans le présent contexte, l observation usuelle d une association directe (et non inversei liant les forces de répulsion et d'attraction cou, de façon équivalente, les flux d'émigration et d'immigrationl d'une meme région.

Le rapprochement de chaque $w_{1}^{A}$ et $w_{1}^{D}$ avec la moyenne geometrique "standardisée" $p_{1}$

$$
P_{1}=\frac{\left[\begin{array}{ll}
\pi & P_{1 t}
\end{array}\right]^{1 / T}}{\left[\begin{array}{ll}
\pi_{k, t} & P_{k t}
\end{array}\right]^{1 / R T}-}
$$

où $F_{k t}$ est la population de la province $k$ au temps $t=$

de la population correspondante figurant dans la colonne mediane du meme tableau révele l'existence d'une association plus ou moins forte entre les parametres de répulsion et d'attraction d'une part et le niveau de la population d'autre part. Ce résultat ne saurait surprendre étant donné la bonne performance usuelle du modele de gravité dans l'etude en coupe transversale de la migration interrégionale.

Pour y voir plus clair, l'on est alors conduit a eliminer l'influence de la taille de la population $P_{1}$ et donc a definir deux indices $\bar{w}_{1}^{A}$ et $\bar{w}_{1}$ exprimant l'intensité par habitant (per capita) des forces de repulsion et dattraction de chaque province:

$$
\bar{w}_{1}^{A}=w_{1}^{A} / p_{1}(11 a) ; \quad w_{1}^{A}=w_{i}^{D} / p_{1}
$$

s (Source: Statistique Canada, années diverses, Catalogue 91-201.) 
TABLEAU 2

Effets principaux lies a l'origine et a la destination

\begin{tabular}{|c|c|c|c|c|c|c|c|c|c|c|}
\hline \multirow{3}{*}{ Province } & \multicolumn{4}{|c|}{ Paranètres } & \multirow{2}{*}{\multicolumn{2}{|c|}{ Population }} & \multicolumn{4}{|c|}{ Paranètres (per capita) } \\
\hline & \multicolumn{2}{|c|}{ Origine $\left(w_{1}^{\hat{A}}\right)$} & \multicolumn{2}{|c|}{ Destination ( $\left.w_{1}^{*}\right)$} & & & \multicolumn{2}{|c|}{ Origine $\left(\bar{n}_{1}^{-}\right)$} & \multicolumn{2}{|c|}{ Destination $\left(\bar{w}_{1}\right)$} \\
\hline & Val eur & Rang & Valeur & Rang & Valeur & Rang & Valeur & Rang & Valeur & Rang \\
\hline Terre-Neuve & 0,403 & 9 & 0,328 & 9 & 0,448 & 9 & 0,900 & 7 & 0,773 & 8 \\
\hline Ile-du-Prince-Edouard & 0,154 & 10 & 0,148 & 10 & 0,097 & 10 & 1,577 & 2 & 1,521 & 3 \\
\hline Nouvelle-Ecosse & 1,155 & 5 & 1,120 & 5 & 0,680 & 7 & 1,699 & 1 & 1,647 & 2 \\
\hline Nouveau-Brunswick & 0,857 & 7 & 0,794 & 7 & 0,553 & 8 & 1,548 & 3 & 1,435 & 4 \\
\hline Québec & 1,584 & 3 & 1,157 & 4 & 5,127 & 2 & 0,309 & 10 & 0,226 & 10 \\
\hline Ontario & 6,168 & 1 & 6,365 & 1 & 6,501 & 1 & 0,949 & 6 & 0,979 & 7 \\
\hline Manitoba & 1,056 & 6 & 0,992 & $b$ & 0,848 & 5 & 1,247 & 5 & 1,171 & 5 \\
\hline Salskatchewan & 0,554 & 8 & 0,568 & 8 & 0,804 & 6 & 0,688 & 9 & 0,706 & 9 \\
\hline Alberta & 1,877 & 2 & 2,578 & 2 & 1,444 & 4 & 1,300 & 4 & 1,786 & 1 \\
\hline Colonbie-Britannique & 1,523 & 4 & 2,164 & 3 & 1,857 & 3 & 0,820 & 8 & 1,165 & 6 \\
\hline
\end{tabular}

La partie droite du tableau 2 qui montre les valeurs prises par ces deux indices (également similaires étant donné leur construction) suggere une classification des dix provinces en quatre groupes distincts:

- un premier groupe de provinces a forte répulsion/attraction (per capita) constitue de trois provinces atlantiques (Ile-du-fince-Edouard, Nouvelle-Ecosse, Nouveau-Brunswick) et de l'Alberta;

- un second groupe limité a la seule province du Manitoba ou $\bar{w}_{1}^{A}$ et $\bar{w}_{1}^{\text {prennent }}$ des valeurs légèrement supérieures a la moyenne;

- un troisieme groupe composé de Terre-Neuve, de l'Ontario et de la ColombieBritannique où les valeurs de $\bar{w}_{1}^{A}$ et $\bar{w}_{1}^{B}$ se situent aux alentours de la moyenne; et

- un quatrième et dernier groupe a faible répulsion/attraction (per capita) où figurent la Saskatchewan et le Québec.

Les valeurs de $\bar{w}_{1}^{A}$ et $\bar{w}_{1}^{B}$ sont particulierement faibles dans le cas du Quebec en raison de la barriere culturelle et linguistique qui sépare cette province du reste du Canada. De fait, elles sont inférieures a la moitié (dans le cas de $\bar{w}_{1}^{A}$ ) et au tiers (dans le cas de $\bar{w}_{1}^{D}$ ) des valeurs enregistrées par la Saskatchewan, l'autre province a faible repulsion/attraction (per capita) alors que les valeurs les plus élevés de $\bar{w}_{1}^{A}$ (pour la Nouvelle-Ecosse) et de $\bar{w}_{1}^{B}$ (pour l'Albertal ne s'établissent qu'a deux fois et demie les valeurs correspondantes relatives a la Saskatchewan. 
Enfin si, comme on l'a remarqué plus haut, les valeurs de $\bar{w}_{1}^{A}$ et $\bar{w}_{1}^{B}$ sont similaires, l'ecart existant entre ces deux valeurs est comparativement plus important dans quatre provinces: a l'avantage de la force de répulsion dans le cas de Terre-Neuve et du Quebec et a l'avantage de la force d'attraction dans le cas de l'Alberta et de Ia Colombie-Britannique.

\section{ASPECTS TEMPORELS}

Les variations annuelles exhibees sur la période 1961-1983 par le phénomene de la migration interprovinciale se traduisent, au niveau de l'effet principal lié au temps, par l'évolution représentée a la figure 1.

\section{FIGURE 1}

Effet principal lié à la variable temps

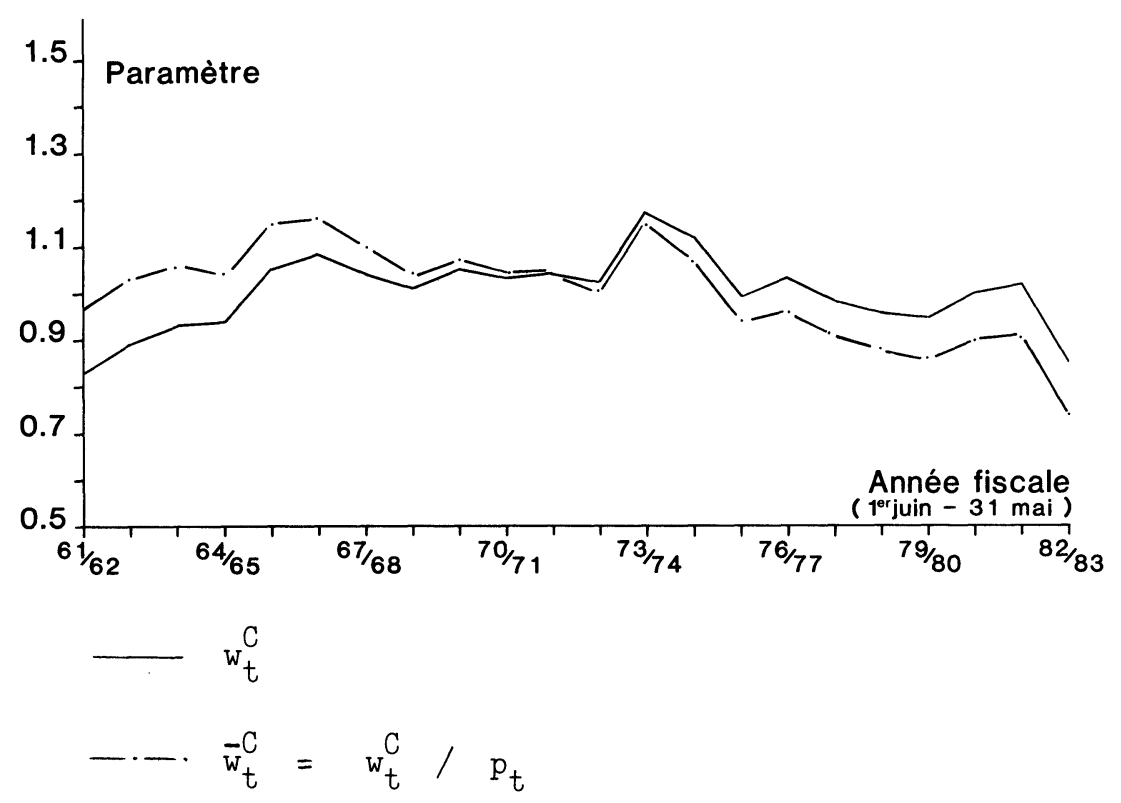

Selon cette figure, la croissance initiale du parametre $w_{t}^{c}$ a fait place, a partir de la periode 1966-1967, a une évolution non monotone marquée par 1) une hausse temporaire peu avant le milieu des années soixante-dix et 2) une décroissance subite lors de la derniere année dobservation. Cependant, cette 
derniere évolution cache une tendance de longue période orientée a la baisse. Mais, comme la pente (négative) de cette tendance est moins prononcée que la pente (positive) de la croissance initialement observée, la valeur de we reste en fin de période supérieure a sa valeur initiale meme si elle s'écarte singulierement de celle-ci lors de la dernière année 1982-1983.

Bien entendu, le paramètre $w_{t}^{c}$ ne reflete que l'intensité globale du phénomene migratoire et donc il importe, comme dans le cas des effets principaux relatifs a l'origine et a la destination, d'eliminer l'effet de taille associé au niveau de la population. L'on est ainsi amené à définir 1 'indice $\bar{w}_{t}^{c}$

$$
\bar{w}_{t}^{c}=w_{t}^{c} / p_{t}
$$

où $p_{t}$ est la moyenne géométrique "standardisée" de la population canadienne au temps $t$

$$
P_{t}=\frac{\sum P_{1 t}}{\left[\begin{array}{ccc}
\pi & (\Sigma & \left.P_{1 t}\right) \\
t & 1
\end{array}\right]^{1 / T}}
$$

L'evolution de cet indice, lequel reflete la propension annuelle de la population canadienne a changer de province de résidence, est similaire a celle du parametre $w_{t}^{c}$. Cependant, la croissance initiale est moins marquée tandis que la tendance a la baisse qui y fait suite est plus forte. Aussi, alors que les niveaux initial et final de $w_{t}^{c}$ sont du meme ordre de grandeur, le niveau final de $\bar{w}_{t}^{c}$ (égal aux $3 / 4$ de son niveau moyen sur la periode entiere dobservation) est-il nettement inférieur a son niveau initial.

Les variations annuelles des parametres wac relatifs a l'effet d'interaction entre la province d'origine $i$ et la variable de temps $t$ sont présentées a la figure 2. Yapparaissent également les variations annuelles des indices $\bar{w}_{1 t}^{A c}$ eliminant 1 'influence du niveau de la population dans la province i

$$
\bar{w}_{1 t}^{A C}=w_{1 t}^{A C} / p_{1 t}
$$

où pit est la moyenne géométrique "standardisée" de la population de la province i au temps $t$

$$
P_{1 t}=\frac{P_{1 t}}{\left[\begin{array}{l}
\pi P_{1 t} \\
t
\end{array}\right]^{1 / T}}
$$


Effet d'interaction entre les variables d'origine et de temps
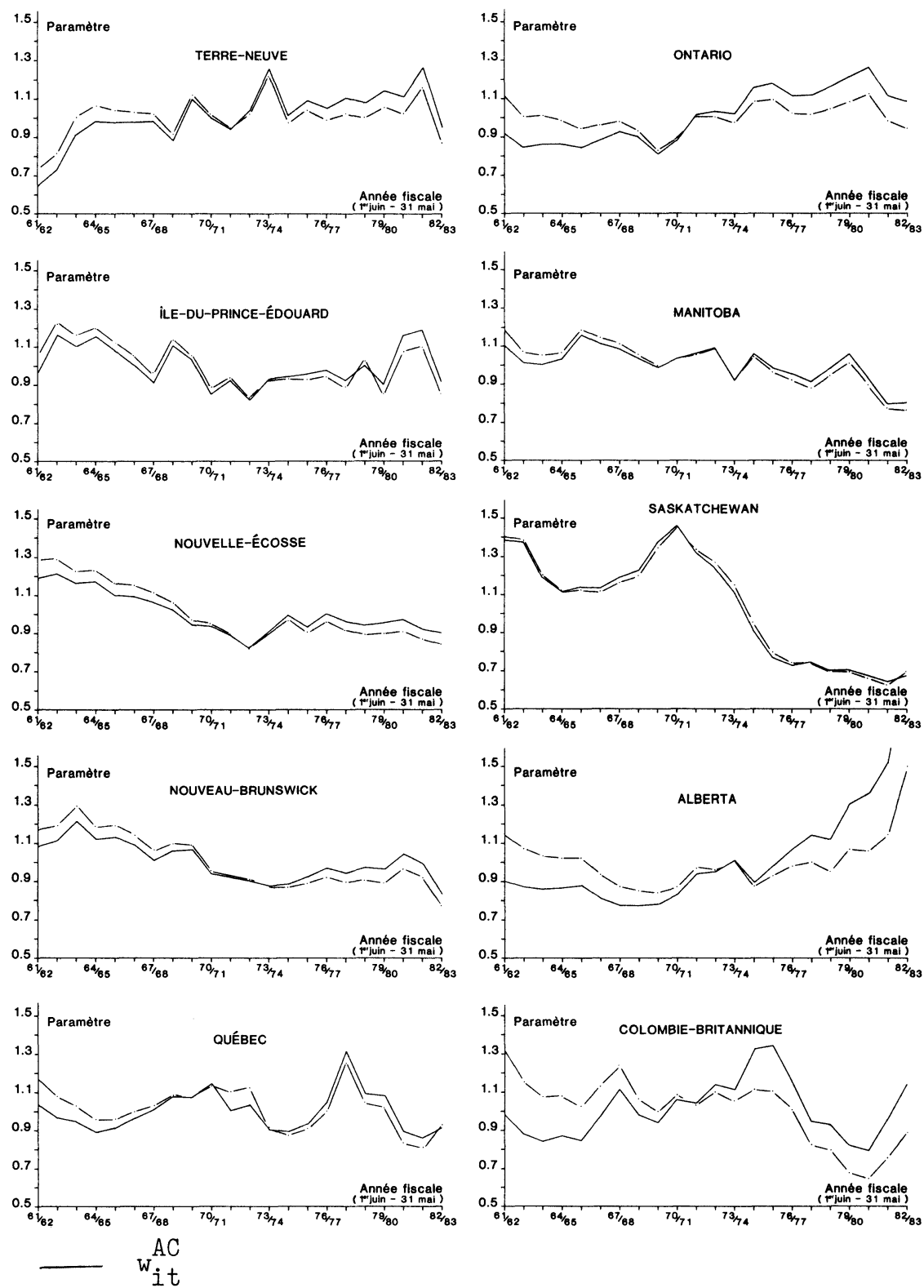

$\cdots \cdot \bar{w}_{i t}^{A C}=w_{i t}^{A C} / p_{i t}$ 
Les variations qui caracterisent l'evolution temporelle de la force de répulsion (valeur absolue et valeur per capita) associée a chaque province sont loin d'etre monotones mais permettent néanmoins la mise en avant de quelques généralisations. Ainsi l'on peut, sur la base des variations de $\bar{w}_{1 t}$, distinguer plusieurs groupes de provinces i:

- un premier groupe de provinces où l'indice wic passe d'une valeur plus élevée dans la première moitié de la période d'observation à une valeur plus faible dans le seconde moitie de cette periode: Ile-du-Prince-Edouard, Nouvelle-Ecosse, Nouveau-Brunswick, Manitoba et Saskatchewan;

- un deuxieme groupe de provinces oú l'indice $\bar{w}_{i t}^{A c}$ présente une évolution inverse a la précedente (valeur plus faible dans la premiere moitié de la période dobservation, valeur plus ellevédans la secondel: Terre-Neuve, ontario et Alberta; et

- un troisieme et dernier groupe de provinces ou l'evolution de wac est cyclique: Québec et Colombie-Eritannique.

L'on remarquera qu'en dépit des evolutions typiques des deux premiers groupes de provinces, la valeur de wac ne s'ecarte genéralement que modérement de sa valeur moyenne. Seules les provinces de la Saskatchewan et de l'Alberta ont un indice $\bar{w}_{1 t}^{A c}$ qui en fin de période est sensiblement différent de celui observé en début de période.

De maniere analogue, les variations annuelles des parametres wec relatifs a l'effet d'interaction entre la province de destination i et la variable de temps t apparaissent a la figure 3 où sont également présentés les variations annuelles des indices $\bar{w}_{1 t}^{D c}=w_{1 t}^{B c} / p_{1 t}$ eliminant l'influence du niveau de la population dans la province i. Ces variations, qui sont typiques de l'évolution temporelle de la force d'attraction ivaleur absolue et valeur per capital associée à chaque province, se pretent a une caractérisation similaire a celle réalisée plus tót en relation avec la force de répulsion. L'on distingue ainsi:

- un premier groupe de provinces où l'indice wo passe d'une valeur plus forte que la moyenne dans la premiere moitié de la periode d'observation a une valeur plus faible dans la seconde moitié Ile-du-Frince-Edouard, Nouvelle-Ecosse, Nouveau-Brunswick, Quebec, Ontario, Manitoba et ColombieBritannique;

- un deuxieme groupe de provinces où wit a subi l'évolution inverse: TerreNeuve et Alberta; et enfin

- un troisieme et dernier groupe de provinces réduit a la seule saskatchewan ou l evolution de $\bar{w}_{1 t}^{B c}$ est de type cyclique.

Far comparaison avec le type precedent d'interaction impliquant la variable temps, ce deuxieme type d'interaction conduit a une moindre stabilite de l'indice pertinent: les variations de we sont plus brusques et d'amplitude sensiblement supérieures a celles de $\bar{w}_{1 t}^{A c}$. Néanmoins, les écarts enregistrés 
Effet d'interaction entre les variables de destination et de temps
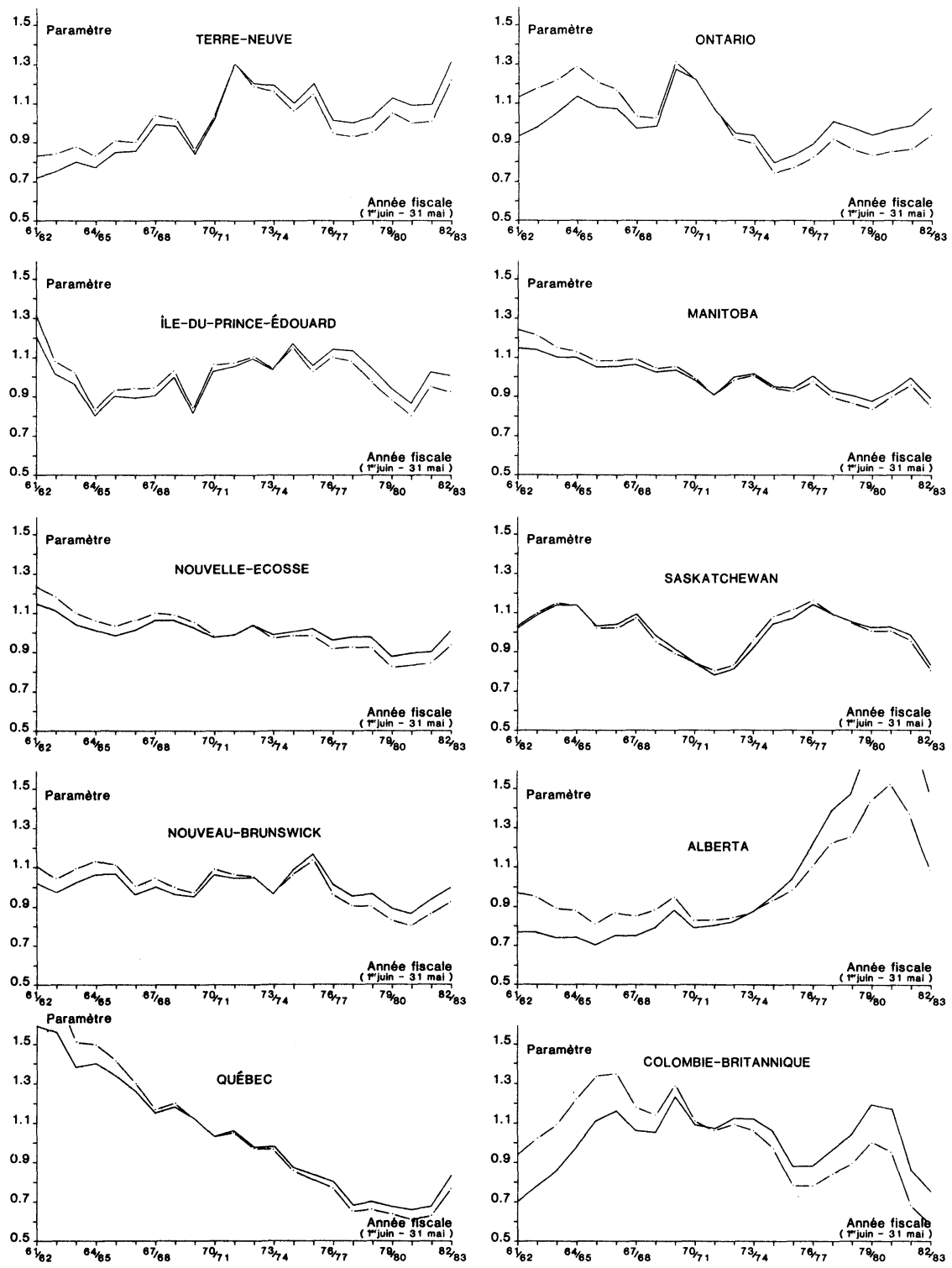

$$
={ }_{w_{j t}^{B C}}^{B C}
$$


pour chaque indice provincial par rapport à la moyenne sont du méme ordre que dans le cas precedent. Aussi, ny a-t-il a nouveau que deux provinces ou l'indice consideré est en fin de periode sensiblement different de sa valeur en début de période. Il s'agit cette fois du Guebec qui a vu son pouvoir d'attraction s'eroder au fil des annés et de l'Alberta qui a vu le sien augmenter fortement dans la seconde partie des annees 1970.

La comparaison des classifications réalisés pour les deux types d eftets ici consideres montre que les pouvoirs de repulsion et dattraction iper capital tendent a evoluer dans le même sens plutôt qu en sens inverse. Mettant de côté les trois provinces ou l'évalution de l'une des deux forces est de type cyclique, QQuebec, Saskatchewan, Colombie-Britannique), l on constate que les variations des indices $\bar{w}_{i t}^{A C}$ et $\bar{w}_{i t}^{8 c}$ sont de meme direction pour six provinces (Ile-du-Frince-Edouard, la Nouvelle-Ecosse, le Nouveau-Brunswick et le Manitoba ou elles sont orientés a la baisse, Terre-Neuve et l'Alberta ou eiles sont orientées à la hausse) alors qu'elles sont de direction inverse pour la seule province d'Ontario.

Au dela des tendances générales dégagés ci-dessus, l analyse des parametres d'interaction entre les variables diespace et de temps se doit detre completée par un examen dordre quantitatif axe sur liacuite des variations enregistrés. A cet effet, le tableau 3 fournit, pour chacun des parametres considerés ainsi que pour chaque population provinciale, Ia valeur d'un indice u, représentatif de son degré de stabilite:

$$
u_{i}=\sum_{t} q_{1 t} \ln \left(q_{1 t} / \hat{q}_{1 t}\right)
$$

où $q_{1 t}$ est la valeur normalisée de $\bar{w}_{i t}^{A C}$, $\bar{w}_{i t}$ ou pit satisfaisant $\sum q_{i t}=1$ et $\hat{q}_{1 t}=1 / T$ est typique d une invariance de la variable considerée.

L'observation de ce tableau suggere tout d'abord une plus grande variabilite des parametres d'interaction impliquant la région de destination que de ceux impliquant la région dorigine, confirmant en cela lune des conclusions issues de l'analyse des variantes non saturés réalisé a la section 1. De plus, le rapprochement des valeurs provinciales correspondantes démontre que les valeurs absolues des parametres dinteraction sont généralement faibles. Il existe néanmoins quelques exceptions notables ayant trait a l'Alberta et a la saskatchewan dans le cas de wac et a l Alberta et au Quebec dans le cas de wac. Mais, ainsi qu'il ressort de la comparaison des indices de stabilité relatifs aux indices $\bar{w}_{1 t}^{A c}$ et wit, la forte instabilité des parametres relatifs a l'Alberta est essentiellement la consequence de l'accroissement démographique que cette province a subi dans les annes soixante-dix. De fait, une fois l'influence de liaccroissement démographique eliminée, seuls l'effet de répulsion relatif a la saskatchewan et 1 effet d'attraction relatif au Quebec se demarquent encore des ensembles correspondants. De façon précise, la valeur de $\bar{w}_{1 t}^{A C} s$ établit a 0,800 pour la Saskatchewan alors qu'elle s'échelonne de 0,053 (Ontario) a 0,343 (Colombie- 
Eritannique) pour les autres provinces; la valeur de $\bar{w}_{i t}$ ac ateint 1,223 pour le Uuebec alors quelle 5 'etage entre 0,102 (Nouveau-Erunswick) et 0,434 (Colombie-Eritannique) pour les autres provinces.

TABLEAU 3

Indices de stabilité relatifs aux paranètres d'interaction entre origine/destination et temps

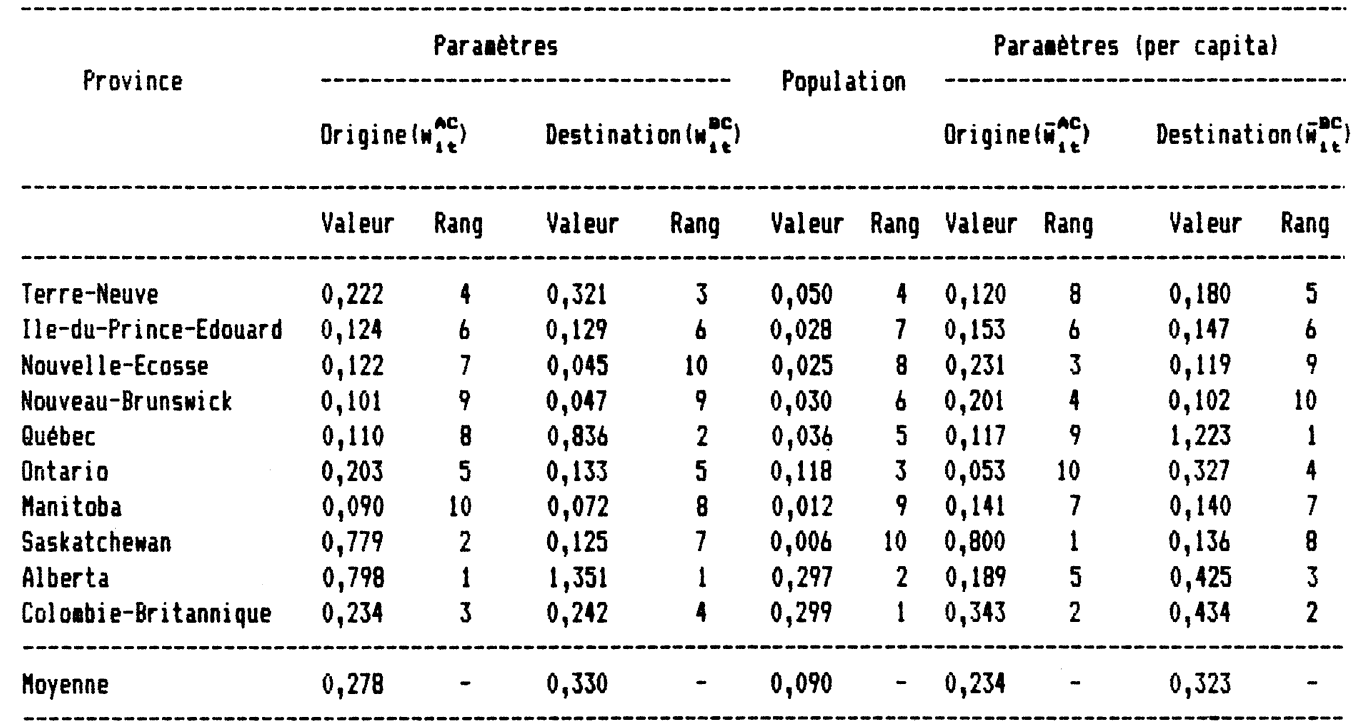

\section{ASPECTS SPATIAUX}

Si le modele log-linéaire permet d'évaluer la stabilité temporelle du phénomene migratoire, il permet aussi de capturer de façon tres réaliste l interaction entre variables d'origine et de destination. A ce propos, il apparait opportun de rappeler ici que les matrices de distances physiques ou de cóuts de transport conventionnellement utilisees pour la mise en oeuvre des modeles de gravité ne réflechissent que trés partiellement les mouvements observes. Ainsi snickars et Weibull (1977) etablissent que la substitution d'une matrice de mouvements précédemment observés pour la matrice des distances physiques conduit a un modele plus performant. Il s'ensuit que la variante du modele log-linéaire ici utilisée, de par son identification des parametres $\bar{W}_{1}^{A B}$ relatifs a l'interaction entre les variables dorigine et de destination, fournit l'occasion d'examiner en quoi la "véritable" structure d'interaction differe de la structure plus normative reposant sur la matrice des distances physiques interprovinciales.

Le tableau 4 présente 1 a matrice estimée des parametres $\bar{w}_{1}^{A} \mathbf{j}$ qui au premier coup doeil apparất etre non-symétrique; d'où l'inégalité des 
distances réelles correspondant aux courants migratoires de directions opposés. De façon plus précise, le calcul, pour chacune des 45 paires de courants inverses, du ratio des parametres d'interaction (en faisant figurer le plus élevé des deux au numérateur) conduit a une valeur moyenne de 1,058 . En d'autres termes, la faculté de se mouvoir entre deux provinces est en moyenne $5,8 \%$ plus elevé dans la direction plus favorable que dans la direction contraire.

TABLEAU 4

Paranetres relatifs a l'interaction entre les variables d'origine et de destination

\begin{tabular}{|c|c|c|c|c|c|c|c|c|c|c|}
\hline \multirow{2}{*}{ Origine } & \multicolumn{10}{|c|}{ Destination } \\
\hline & T. $-N$. & I. $-P .-E$. & N. $-E$. & N. $-B$. & QUE. & ONT. & MAN. & SASK. & ALB. & C. $-B$. \\
\hline Terre-Neuve & - & 1,715 & 2,560 & 1,759 & 1,317 & 1,487 & 0,610 & 0,420 & 0,618 & 0,418 \\
\hline Ile-du-Prince-Edouard & 1,786 & - & 3,722 & 3,446 & 0,766 & 0,927 & 0,570 & 0,451 & 0,565 & 0,423 \\
\hline Nouvelle-Ecosse & 2,626 & 3,865 & - & 2,644 & 0,957 & 0,943 & 0,453 & 0,361 & 0,419 & 0,603 \\
\hline Nouveau-Brunswick & 1,765 & 3,253 & 2,618 & - & 2,413 & 0,953 & 0,495 & 0,351 & 0,414 & 0,403 \\
\hline Québec & 1,297 & 0,803 & 0,902 & 2,293 & - & 2,306 & 0,699 & 0,499 & 0,667 & 0,866 \\
\hline Ontario & 1,522 & 1,001 & 0,900 & 0,968 & 2,039 & - & 0,950 & 0,721 & 0,658 & 0,821 \\
\hline Manitoba & 0,592 & 0,541 & 0,432 & 0,457 & 0,766 & 0,923 & - & 5,867 & 1,832 & 2,080 \\
\hline Saskatchewan & 0,380 & 0,414 & 0,360 & 0,339 & 0,498 & 0,714 & 6,238 & - & 6,061 & 3,863 \\
\hline Alberta & 0,605 & 0,562 & 0,460 & 0,419 & 0,622 & 0,639 & 1,710 & 5,512 & - & 4,079 \\
\hline Col oubie-Britannique & 0,450 & 0,458 & 0,690 & 0,433 & 0,888 & 0,831 & 1,810 & 3,582 & 3,396 & - \\
\hline
\end{tabular}

Au dela de cette valeur moyenne, l'éventail des valeurs prises par les 45 ratios calculés se caracterise par une distribution relativement homogene entre 1 et 1,1 alors que seuls 5 ratios dépassent la valeur 1,1: voir le tableau 5. De plus, la liste des paires de provinces pour lesquelles l'asymetrie des termes relationnels est comparativement plus élevée indique qu'il est plus facile de se rendre:

- des Praries (Manitoba, Saskatchewan, Alberta) vers la Colombie-Britannique

- de la Colombie-Britannique vers les trois provinces les plus a 1 'est (Terre-Neuve, Ile-du-Prince-Edouard, Nouvelle-Ecosse) et

- du Québec vers l'Ontario

que dans les directions opposés.

L'on sait que, toutes choses étant égales par ailleurs, la difficulté de se déplacer dans l'espace augmente avec la distance. Aussi, vu que l'ordre des provinces utilisé dans cet article reflete leur position géographique d'est en ouest dans le systeme canadien, l'on doit s'attendre a ce que la valeur des parametres d'interaction apparaissant au tableau 4 diminue lorsque 1 'on se 
déplace, dans le triangle supérieur ic'est-a-dire au-dessus de la diagonale principalel, de gauche a droite le long d'une ligne ou de bas en haut le long d'une colonne et, dans le triangle inferieur, dans les directions opposeses. Or tel n'est pas toujours le cas, ce qui conduit a conclure que les distances rélles entre provinces sont loin de refleter les distances physiques. Une lecture attentive du tableau 4 indique, en particulier, que l'importance de lobstacle spatial dans les mouvements de chaque province atlantique vers 1 'ouest du pays n'augmente pas de façon monotone avec le deplacement vers l'ouest de la province de destination. De fait, au lieu de diminuer, le parametre d'interaction se releve si l'on substitue l'Alberta a la Saskatchewan comme province de destination, revenant au niveau de la valeur estime dans le cas ou le Manitoba est la région de destination.

TABLEAU 5

Résumé des valeurs prises par les quotients des parametres d'interaction relatifs aux courants migratoires en sens opposés

\begin{tabular}{|c|c|c|c|c|}
\hline Intervalles & Nombre & Courat & & Valeurs \\
\hline 1,075 a 1,000 & 5 & $\begin{array}{l}\text { Alberta } \\
\text { Manitoba } \\
\text { Colombie-Britannique } \\
\text { Québec } \\
\text { Terre-Neuve } \\
\text { Saskatchewan } \\
\text { Alberta } \\
\text { Manitoba } \\
\text { Ile-du-Prince-Edouard } \\
\text { Nouveau-Brunswick } \\
\text { Colombie-Britannique } \\
\text { Quebec } \\
\text { Saskatchewan } \\
\text { Colombie-Britannique }\end{array}$ & $\begin{array}{l}\text { Colombie-Britannique } \\
\text { Colombie-Britannique } \\
\text { Nouvelle-Ecosse } \\
\text { Ontario } \\
\text { Saskatchewan } \\
\text { Alberta } \\
\text { Nouvelle-Ecosse } \\
\text { Québec } \\
\text { Saskatchewan } \\
\text { Manitoba } \\
\text { Ile-du-Prince-Edouard } \\
\text { Ile-du-Prince-Edouard } \\
\text { Terre-Neuve } \\
\text { Terre-Neuve }\end{array}$ & $\begin{array}{l}1,201 \\
1,149 \\
1,145 \\
1,131 \\
1,105 \\
1,100 \\
1,098 \\
1,096 \\
1,091 \\
1,084 \\
1,082 \\
1,081 \\
1,079 \\
1,077\end{array}$ \\
\hline $\begin{array}{l}1,050 \text { a } 1,075 \\
1,025 \text { a } 1,050 \\
1 \text { a } 1,025\end{array}$ & $\begin{array}{r}9 \\
10 \\
12\end{array}$ & & & \\
\hline
\end{tabular}


Lon remarquera aussi que si l'Alberta exerce sur les habitants des provinces atlantiques une attraction comparativement plus forte que ne le laisse supposer sa position géographique dans le système canadien, liontario exerce une influence similaire sur les habitants de Terre-Neuve et de l'Ile-duPrince-Edouard et de même la Colombie-Britannique sur les habitants du Québec et de 1 'Ontario.

Vu les observations précedentes, il apparaît souhaitable déclairer ci-apres les écarts existant entre les distances interprovinciales reelles dune part et physiques d'autre part. A cet effet, une procedure originale en deux etapes successives est utilisee qui, en fin de compte, fournit, pour chaque paire origine-destination, une valeur estimee de la distance rélle directement comparable à la distance physique.

La premiere étape de cette procédure consiste a évaluer l'influence générale exercée par la distance dans la migration interprovinciale. Si lon suppose que cette influence peut etre représentée par une fonction puissance de la distance

$$
t_{i j} \div e^{-h d_{1 j}}
$$

où d, est la distance physique entre la ville principale de la province i et celle de la province $j$ et $h$ est un parametre positif ou nul a determiner. Il importe tout d'abord de fixer tis de telle sorte qu'il soit directement comparable avec le parametre d'interaction correspondant was. Cieci conduit alors à définir

$$
t_{1 j}=k u_{1} e^{-h d_{1 j} v_{j}}
$$

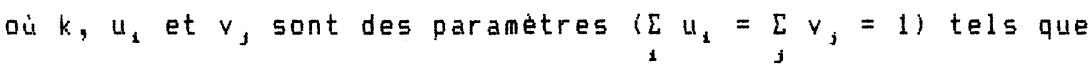

$\pi_{i \neq j} t_{i j}=\pi_{j \neq i} t_{i j}=1$

Il est clair que, pour toute valeur donné de h, l'estimation de ces parametres peut 5 effectuer tres simplement a partir de formules etablies sur la base d'une méthodologie similaire a celle utilisée plus haut pour l'estimation des parametres d'interaction relatifs a la variante de quasi-independance du modele log-linéaire lles details de cette methodologie peuvent etre fournis par 1 auteur sur demande).

Four obtenir la valeur du terme relationnel t, , refletant le seul effet de la distance physique entre les provinces i et $j$, il suffit donc d'estimer la valeur optimale $h^{*}$ de l'exposant $h$. Ici, cette valeur est definie comme celle qui minimise l ecart entre les matrices $w=\left(w_{i j}^{A B}\right)$ et $t=\left(t_{1 j}\right)$ sur la base de 1 indice de type $X^{2}$ suivant:

$$
I=\sum_{j, 1} \sum \frac{\left(t_{1},-W_{1 J}^{A B}\right)^{2}}{W_{1 j}^{A B}}
$$


Sa determination pratique est realisée sur la base dune application de la methode Faphson-Newton a la recherche du minimum de I(h): pour les détails d'une telle application, voir Ledent (1980: 340). Tous calculs faits, il ressort que $h^{*}=0,8884$.

Dès lors, si di, dénote la distance réelle de i à j, la relation

$$
\frac{W_{i j}^{A B}}{t_{i j}}=\left[\frac{d_{i j}^{\prime}}{d_{i j}}\right]^{-n}
$$

permet d'aboutir a l'estimation des distances rélles dis

$$
y_{i j}=A_{17}\left[\begin{array}{l}
p^{1}, j \\
w_{1}^{A B}
\end{array}\right]^{-1 / n}
$$

[lesquelles, 1 on remarquera au passage, sont telles que $\pi_{i \neq j} d_{i j}=\pi_{i j} d_{i j}$ et $\left.\pi_{j \neq 1} d_{i j}=\pi_{j \neq 1} d_{i j}\right]$.

Le tableau b montre les distances rélles obtenues sur la base de (21), en valeur absolue aussi bien que comme fractions des distances physiques correspondantes. Le quotient $d_{i j}^{\prime} / d_{1}$, est généralement inferieur a 1 pour tout déplacement entre deux provinces situées d'un meme côté de l'Ontario (que ce soit un deplacement entre deux provinces atlantiques ou entre deux provinces de 1 ouest canadienl mais superieur a 1 pour tout deplacement entre deux provinces situees de part et d'autre de l'Ontario (qu'il ait lieu d'est en ouest ou dans la direction opposée). Une telle observation nous incite donc a croire que 1 élasticité de la propension a migrer n'est pas, comme le suppose (17), homogene dans l'espace mais qu'elle tend a diminuer plus rapidement quand la distance s'accroint.

Nonobstant la fragilité de l'expression (17), le tableau b nous indique qu en maintes occasions la distance reelle differe substantiellement de la distance physique. En particulier, elle est trés inférieure à cette derniere en ce qui concerne les mouvements enregistres entre Terre-Neuve et 1 : Ontario, la Nouvelle-Ecosse et l'Ontario, le Québec et le Nouveau-Brunswick, le Manitoba et la Saskatchewan, la Saskatchewan et la Colombie-Britannique. Par contre, la relation est largement inversée dans le cas des mouvements entre Terre-Neuve et la Saskatchewan, Terre-Neuve et la Colombie-Britannique, I'Ile-du-PrinceEdouard et Quebec. D'une façon genérale, ces écarts substantiels entre distances physiques et rélles correspondent, pour les premiers, a des relations privilégiés et, pour les seconds, de des relations peu développés.

Lon peut sans nul doute pousser plus avant l'analyse de la dimension spatiale, mais lon se garde ici d'une telle tentation dans le mesure où 
TABLEAU 6

Distances interprovinciales: valeurs physiques et reelles (en ailles)

Destination

Origine T.-N. I.-P.-E. N.-E. N.-B. Que. Ont. Man. Sask. Alb. C.-B.

1. Valeurs physiques

Terre-Neuve

Ile-du-Prince-Edouard

Nouvelle-Ecosse

Nouveau-Brunswick

Quebec

Ontario

Manitaba

Saskatchewan

Alberta

Colombie-Britannique

\section{$\pm$}

900

934

1073

1617

1952

3113

3628

3956

4600

$\begin{array}{cc}900 \\ * \\ 174 \\ 201 \\ \\ 745 \\ 1080 \\ 2241 \\ 2756 \\ 3084 \\ 3 & 728\end{array}$

\begin{tabular}{|c|c|}
\hline 934 & 1073 \\
\hline 174 & 201 \\
\hline * & 192 \\
\hline 192 & 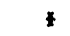 \\
\hline 776 & 584 \\
\hline 1111 & 919 \\
\hline 2272 & 2080 \\
\hline 2787 & 2595 \\
\hline 3115 & 2923 \\
\hline 3759 & 3567 \\
\hline
\end{tabular}

$\begin{array}{llllll}1617 & 1952 & 3 & 113 & 3628\end{array}$

$745 \quad 1080 \quad 2241 \quad 2756$

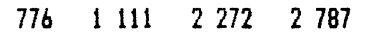

$\begin{array}{lllll}584 & 919 & 2080 & 2595\end{array}$

$335 \quad 1496 \quad 2011$

$+13041819$

$1496 \quad 1304$

1304

515

$2011 \quad 1819$

$2339 \quad 2147$

2983279

2. Valeurs réelles

$\begin{array}{lccccccccccccc}\text { Terre-Neuve } & * & 840 & 542 & 780 & 1243 & 1226 & 3943 & 6148 & 4477 & 9973 \\ \text { Ile-du-Prince-Edouard } & 902 & * & 173 & 178 & 1110 & 1013 & 2068 & 2750 & 2400 & 4770 \\ \text { Nouvelle-Ecosse } & 527 & 165 & * & 242 & 876 & 1006 & 2711 & 3578 & 3410 & 3247 \\ \text { Nouveau-Brunswick } & 777 & 189 & 245 & * & 291 & 938 & 2315 & 3492 & 3260 & 4820 \\ \text { Québec } & 1264 & 1052 & 935 & 309 & * & 399 & 1805 & 2703 & 2192 & 2343 \\ \text { Ontario } & 1194 & 928 & 1060 & 921 & 458 & * & 1446 & 2018 & 2517 & 2813 \\ \text { Manitotia } & 4079 & 2190 & 2861 & 2533 & 1628 & 1493 & * & 225 & 938 & 1166 \\ \text { Saskatchewan } & 6977 & 3032 & 3589 & 3622 & 2707 & 2039 & 210 & * & 250 & 594 \\ \text { Albertal } & 4588 & 2418 & 3069 & 3213 & 2371 & 2598 & 1013 & 278 & * & 629 \\ \text { Colombie-Britannique } & 9173 & 4365 & 2788 & 4447 & 2276 & 2774 & 1364 & 647 & 773 & *\end{array}$

3. Ratios valeur réelle/valeur physique

$\begin{array}{lcccccccccc}\text { Terre-Neuve } & * & 0,933 & 0,580 & 0,727 & 0,769 & 0,628 & 1,267 & 1,695 & 1,132 & 2,168 \\ \text { Ile-du-Prince-Edouard } & 0,892 & * & 0,992 & 0,883 & 1,490 & 0,938 & 0,923 & 0,998 & 0,788 & 1,279 \\ \text { Nouvelle-Ecosse } & 0,564 & 0,950 & * & 1,262 & 1,128 & 0,905 & 1,193 & 1,284 & 1,095 & 0,864 \\ \text { Nouveau-Brunswick } & 0,724 & 0,942 & 1,276 & * & 0,499 & 1,020 & 1,113 & 1,346 & 1,115 & 1,351 \\ \text { Queber } & 0,782 & 1,413 & 1,205 & 0,529 & \star & 1,190 & 1,207 & 1,344 & 0,937 & 0,786 \\ \text { Ontario } & 0,612 & 0,859 & 0,954 & 1,002 & 1,368 & \star & 1,109 & 1,109 & 1,172 & 1,008 \\ \text { Manitoha } & 1,310 & 0,977 & 1,259 & 1,218 & 1,088 & 1,145 & * & 0,437 & 1,113 & 0,841 \\ \text { Saskatchewan } & 1,896 & 1,100 & 1,288 & 1,396 & 1,346 & 1,121 & 0,408 & * & 0,761 & 0,570 \\ \text { Albertat } & 1,160 & 0,784 & 0,985 & 1,099 & 1,014 & 1,210 & 1,202 & 0,847 & * & 0,814 \\ \text { Colombie-Britannique } & 1,994 & 1,171 & 0,742 & 1,247 & 0,763 & 0,994 & 0,983 & 0,621 & 1,000 & *\end{array}$

Source: Valeurs physiques: Ternote et Frechette (1979). 
- I estimation des donnés migratoires interprovinciales par statistique canada repose sur une ventilation contestable des migrations par province de destination (voir statistique Canada 1977, catalogue 92-208, pp. 17-18),

- la distance physique entre deux provinces n'est pas necessairement refieté de façon adéquate par la distance routiere entre les villes principales de ces provinces.

\section{CONCLUSION}

Lapplication du modele log-lineaire aux courants annuels de migration interprovinciale enregistrés depuis $15 t i$ a permis declairer les aspects spatio-temporeis de ce phenomene. D'un point de vue spatial, l'on a demontré la forte interaction existant entre régions d'origine et de destinationi un résultat qui, on l'espere, incitera un plus grand nombre de chercheurs a privilegier les courants migratoires origine-destination au detriment des flux bruts régionaux.

D'un point de vue temporel, l'on a entre autres mis en évidence une tendance longue a la baisse de la propension a changer de province ainsi qu'une plus grande variabilite de l'effet dattraction que de leffet de répulsion relatifs a chaque province. De plus, ces deux effets tendent a varier dans le meme sens plutót qu'en sens contraire.

Four conclure, l'on signale que l'analyse ci-dessus peut être completée par une analyse econometrique ayant pour but de relier les variations des parametres subissant l'influence de la variable temps aux changements observes dans l'environnement socio-économique pertinent. Ainsi que le suggere le travail réalisé par Ledent (1984),

- le parametre we est significativement influencé par les variations du taux de chomage (impact négatif) et du taux de salaire hebdomadaire (impact positif) observés au niveau national,

- les parametres WA et wit sont significativement influences par les variations locales des memes variables.

\section{Annexe}

Détermination des parametres relatifs à la variante

de quasi-indépendance

Les parametres relatifs a la variante de quasi-indépendance ont été

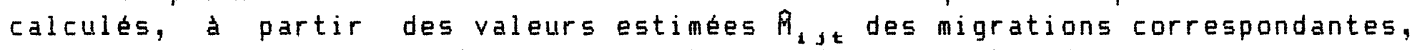
par l application successive des formules ci-dessous ila démonstration de ces formules peut etre obtenue de l'auteur sur demande). 
1. Interaction des variables d'origine (destination) et de temps:

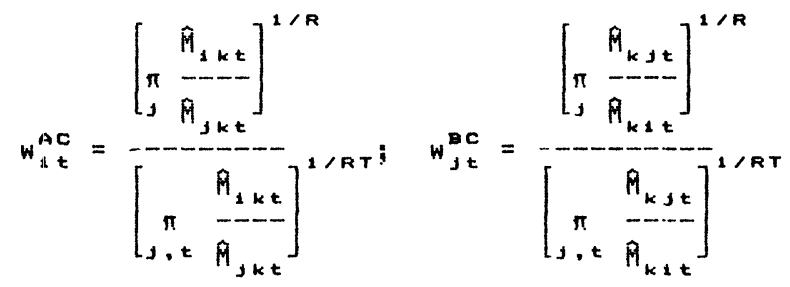

(où $k$ est arbitrairement choisi).

2. Effet principal lié au temps:

$$
\left.w_{t=}^{\prime}=\frac{-e_{t}}{\left[e_{t}\right.}-\overline{e_{t}}\right]_{1} / T
$$

avec $e_{t}=\frac{M_{1, t}}{w_{1 t}^{A C} W_{j t}^{B C}}$ (ou $j$ et j sont arbitrairement choisis).

3. Effets principaux liés à l'origine et au temps:

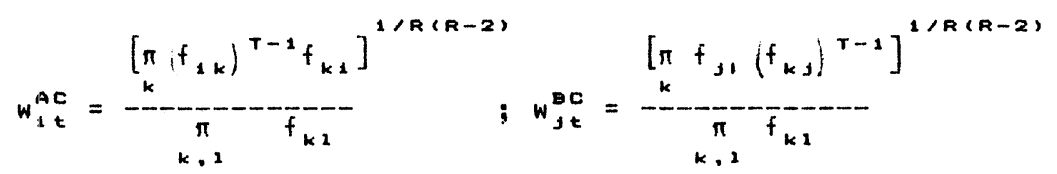

avec $f_{13}=\frac{\hat{P}_{1, t}}{W_{t}^{C} W_{i t}^{A C} W_{j t}^{B C}}$ (où t est arbitrairement choisi).

4. Effet général moyen:

$$
W=\left[\begin{array}{ll}
\pi & g_{k}
\end{array}\right]^{1 / R}
$$

avec $g_{k}=\frac{\hat{A}_{1 k t}}{W_{1}^{A} W_{k}^{B} W_{t}^{C} W_{1 t}^{A C} W_{k t}^{B C}}$ lù i et $t$ sont arbitrairement choisis) ou bien

$$
g_{k}=\frac{B_{k y t}}{W_{k}^{A} W_{j} W_{t}^{C} W_{k t}^{A C} W_{j t}^{C C}} \text { (ou j et } t \text { sont arbitrairement choisis). }
$$


5. Interaction des variables d'origine et de destination:

$$
W_{1 J}^{A B}=\frac{\hat{M}_{1 j t}}{W_{1}^{A} W_{J}^{B} W_{t}^{C} W_{1 t}^{A C} W_{J t}^{B C}} \text { (out } t \text { est arbitrairement choisi). }
$$

Bien entendu, les produits apparaissant dans chacune des formules ci-dessus excluent les courants estimés H $_{1}$ te tels que $j=i$.

\section{RÉFÉRENCES BIBLIOGRAPHIQUES}

BAYDAR, N., 1983. Analysis of the Temporal stability of lligration Patterns in the Context of Hultiregional Forecasting. Voorburg, The Netherlands, Netherlands Interuniversity Demographic Institute. (Working Paper 38)

BISHOP Y.M., FIENBERG S.E. et HOLLAND R.W., 1975. Discrete Nultivariate Analysis: Theory and Practice. Cambridge, Massachussets, M.I.T. Press.

DEMING, W. et STEFHAN, F., 1940. "On a Least Squares Adjustment of a Sampled Frequency Table when Expected Marginal Totals are known". Annals of Nathe atical statistics, 11, 427-444.

DIXON, W.J. et BROWN, M.B., 1979. BHDP-T9. Bionedical Computer Programs P-series.. Berkeley, California, University of California Fress.

FIENBERG, S.E., 1977. The Analysis of Cross-Classified Categorical Data. Cambridge, Massachussets, M.I.T. Press.

HUA, C.I. et FORELL, F., 1979. "A Critical Review of the Development of the Gravity Model". International Regional Science Review, 4, 2, 97-126.

LEDENT, J., 1980. "Calibrating Alonso's General Theory of Movement: The Case of Interprovincial Migration Flows in Canada". Sisteni Urbani, 2/3, 327-358.

LEDENT, J., 1984. "Demoeconomic Modeling of Interprovincial Migration in Canada: The Longitudinal Case". Faper Presented at the 8th Annual Meeting of the Canadian Regional Science Association. Guelph, Ontario, May 31- June 1 .

Nofíis, D., 1983. "New Sources of Canadian Small Area Migration Data". Review of Public Data Use, 11, 1, 11-25.

SNICKARS, F. et WEIEULL, J.W., 1977. "A Minimum Information Principle. Theory and Practice". Regional Science and Urban Econonics, 7, 137-168.

STATISTIQUE CANADA, 1979. Projections deographiques pour le canada et les provinces 1976-2001. Catalogue 91-514 (hors serie). Ottawa, Information Canada.

STATISTIQUE CANADA, (années diverses). Estimations de population pour le Canada et les provinces. Catalogue 92-201 (annuel). Ottawa, Information Canada. 
STATISTIQUE CANADA, (années diverses). Les igrations internationales et interprovinciales au Canada. Catalogue 91-208 (annuel). Ottawa, Information Canada.

TERMOTE, M. et FRECHETTE, R.. 1979. Les variations du courat igratoire interprovincial. Montréal, Institut national de la recherche scientifique Urbanisation.

U.S. Bureau of the Census, 1967. "Revised Projections of the Population of States: 1970 to 1985". Current Population Reports. Series P-25, no 375. Washington, D.C., U.S. Government Printing Office.

WILLEKENS, F., 1982. "Multiregional Population Analysis with Incomplete Data". in K. Land et A. Fogers, eds. Multidinensional Hatheatical Denography. New York, N.Y., Academic Press, 43-111.

WILLEKENS, F., 1983a. "Log-Linear Modeling of Spatial Interaction". Papers of the Regional Science Association, 52, 187-205.

WILLEKENS, F., 1983b. "Specification and Calibration of Spatial Interaction Models. A contingency Table Ferspective and an Application to Intraurban Migration in Rotterdam". Tijdschrift voor Economische en Sociale Geografie, $74,4,239-252$. 


\section{RÉSUHÉ - SUMHARY - RESUMEN}

\section{LEDENT Jacques - Une analyse log-lintaire des courants aigratoires interprovinciaux: Canada, 196!-1983}

Cet article présente une analyse approfondie des caractéristiques spatio-teaporelles de la aigration interprovinciale au Canada réalisée sur la base de données langitudinales tirées du fichier des allocations faniliales. La méthodologie utilisée fait appel au aodèle log-linéaire sous-jacent à la notion de tableau de contingence, lequel modele autorise une représentation siaple des données observées a l'aide de paranètres caractéristiques des diverses interactions possibles entre les variables concernées (variables d'origine, de destination et de teups dans le cas present). Dans une preaiere étape, un examen comparé de la performance des diverses variantes non saturées (variantes où anquent un ou plusieurs des parametres pertinents) permet de clarifier l'influence globale de chacune des variables. Dans une deuxième étape, cette influence est précisée a l'aide d'une investigation détaillée des paraaetres relatifs à la variante de quasi-independance, c'est-d-dire, la variante où seul l'effet d'interaction s'exerçant entre les trois variables est ignoré. Outre la suggestion que les variables d'origine et de destination ne sont pas indépendantes (résultat qui confirae la nécessité de mettre l'accent sur les courants igratoires dans l'étude de la migrationl, cette investigation en avant quelques conclusions sur les variations teaporelles de la aigration interprovinciale.

\section{LEDENT Jacques - A Log-linear Analysis of Interprovincial Streans of Migrations Canade, 1961-1983}

This article presents an in-depth analysis of spatial-teoporal characteristics of interprovincial aigration in Canada carried out with longitudinal data obtained fron the fanily allowance file. The aethodology used calls on the log-linear model underlying the notion of the contingency table. This nodel pernits a siaple representation of the observed data, with the aid of paraneters typical of the any possible interactions between the variables in question fvariables of origin, destination and period in the present casel. First, a comparative examination of the performance of several unsaturated variants (variants where one or aore pertinent parameters are aissing) allows clarification of the overall influence of each variable. Second, this influence is further specified with the aid of a detailed investigation of the paraneters that are relative to the quasi-independence variance, that is to say, the variance where only the effect of interaction between the three variables is ignored. Together with the suggestion that the variables of origin and destination are not independent la result that confirms the need to emphasize interregional streans in the study of aigrationl, this investigation sets forth some conclusions about the temporal variations of interprovincial nigration. 


\section{LEDENT Jacques - Un análisis logo-lineal de los flujos aigratorios interprovinciales: Canadá, 1961-1983}

El presente artículo ofrece un análisis ainucioso de las caracteristicas espaciales y teaparales de la aigración entre las provincias de Canadá, sustentandose en datos de carácter longitudinal provenientes del archivo de Subsidios a la Familia. La metodología que se llevó a cabo está basada en el modelo logo-lineal subyacente a la noción de tabla de contingencia, dicho modelo peraite elaborar una representación siaple de los datos observados con ayuda de parámetros caracteristicos de las diversas interacciones que se dan entre las variables en juego (variables de origen, de destino y de tieapo, en este estudiol. En una priøera etapa, se hace un examen coaparativo de la acción de las diversas variantes no saturadas (variantes en donde faltan uno o varios de los paránetros pertinentes) 10 que permite esclarecer la influencia glabal de cada una de las variables. En una segunda etapa, esta influencia es especificada gracias al estudio pormenorizado de los parámetros relativos a la variante de casi-independencia, o sea la variante en donde únicamente el efecto de interacción que se da entre las tres variables no se toma en cuenta. Aparte de sugerir que las variables de origen y de destino no son independientes (resultado que confirma la necesidad de hacer hincapié en el estudio de las corrientes aigratorias al tratar la migraciónl, la presente investigación sienta algunas conclusiones acerca de las fluctuaciones teaporales de la agración interprovincial. 